\title{
Belegarzttarif im Bereich UV/MV/IV
}

\section{U. Wanner}

Korrespondenz:

Schweizerische Belegärzte-

Vereinigung

Moosstrasse 2

CH-3073 Gümligen

Tel. 0319527905

Fax 0319527683

info@svbp.ch
Dieser Tage haben Vertreter der Medizinaltarifkommission und der Schweizerischen Belegärzte-Vereinigung den neuen Vertrag betreffend Belegarzttarif im Bereich UV/MV/IV unterzeichnet. Der Tarif regelt die Belegarzthonorare für ambulante und stationäre Spitalbehandlungen gemäss UV/MV/IV mittels indikationsbezogener Arztpauschalen; er kommt in Spitälern zur Anwendung, an denen Teilpauschalen für die Behandlung von UV-/MV-/IV-Patienten vereinbart sind.

Ausgangspunkt der Diskussionen über die Vergütung von belegärztlichen Leistungen war der Umstand, dass der TARMED die belegärztliche Struktur nur ungenügend berücksichtigt. Seit April 2004 gilt daher für Belegärzte ein Zusatzabkommen zum TARMED-Rahmenvertrag, der im UV-/MV-/IV-Bereich neben der Abgeltung der invasiven Leistungen, die im Spital von Belegärzten erbracht werden, auch eine Praxiskostenabgeltung für diese Zeit vorsieht. Dieses Zusatzabkommen war eine vorübergehende Lösung und wird nun durch den neuen Vertrag abgelöst, der per 1. Januar 2007 in Kraft tritt.

Ziel des neuen Vertrages ist es, die häufigsten belegärztlichen Leistungen im Spital durch sogenannte indikationsbezogene Arztpauschalen (IAP) zu erfassen. Der Belegarzt wird den Versicherern im UV-/MV-/IV-Bereich zukünftig nicht mehr Rechnung mit Einzelleistungen gemäss TARMED stellen, sondern anhand der medizinischen Indikation (Diagnose) eine Pauschale verrechnen.

Die indikationsbezogenen Arztpauschalen wurden in enger Zusammenarbeit mit den betroffenen Fachgesellschaften ermittelt. Bis zum heutigen Zeitpunkt sind rund 110 derartige IAP erstellt. Es handelt sich schwergewichtig um Pauschalen in Bereichen der Orthopädie, Neurochirurgie, Handchirurgie und ORL. Alle definierten Pauschalen beinhalten auch einen Pauschalbetrag an den Anästhesisten, sofern dieser Belegarzt ist.

Die Liste dieser IAP ist auf der Website der Schweizerischen Belegärzte-Vereinigung (www. svbp.ch) aufgeführt.
Der Belegarzttarif ist mit Inkrafttreten per 1. Januar 2007 nicht ein kompletter, abgeschlossener Katalog von Pauschalen, sondern soll in den nächsten Monaten laufend ergänzt werden. Schon im Vertragstext ist eine Evolutivklausel enthalten; Vorstand SBV wie auch UV/MV/IV wollen den eingeschlagenen Weg weitergehen und sowohl in weiteren Fachrichtungen als auch innerhalb der schon behandelten Fachgebiete weitere Pauschalen entwickeln.

Um diesem Belegarzttarif beitreten zu können, muss der interessierte Belegarzt eine Selbstdeklaration ausfüllen (Formular ist beim Sekretariat SBV oder auf der Website der SBV, www.svbp.ch, erhältlich) und eine Bestätigung der selbständigen Erwerbstätigkeit (seitens der AHV-Ausgleichskasse) beibringen. Mitglieder der SBV senden diese Unterlagen an das Sekretariat SBV, Nichtmitglieder an die MTK, Fluhmattstrasse 1, 6002 Luzern.

Für die SBV-Mitglieder sind Anschluss- und jährliche Konzessionsgebühren im Mitgliederbeitrag inbegriffen; für Nichtmitglieder SBV beträgt die einmalige Anschlussgebühr Fr. 900.und die jährliche Konzessionsgebühr Fr. 250.-.

Eine paritätische Vertrauenskommission - für Streitfälle - und eine paritätische Tarifkommission - für Vollzug und Weiterentwicklung des Tarifvertrages - bilden wichtige Elemente dieser Tarifpartnerschaft, die ohne Zweifel im Interesse von Patienten, Leistungserbringern und Versicherungen liegt.

Die Vertreter der vier Vertragsparteien begrüssen das Zustandekommen des Vertrages, zumal die Belegärzte gerade im Unfallversicherungsbereich ein wichtiger Partner sind. Zudem enthält der Vertrag auch verbindliche Qualitätsparameter, wie Dauer der Arbeitsunfähigkeit, Zugang zu den Spitälern und die Beobachtung von Komplikationen.

Für nähere Auskünfte stehen MTK (A. Weissenburger) und SBV (Sekretariat, Dr. Wanner oder C. Lüssi) gerne zur Verfügung. 